\title{
PROBLEMÁTICA DA ORIENTAÇÃO DO PACIENTE SOB CONTROLE DE DIURESE
}

\author{
Brigitta E. P. Castellanos* \\ Célia P. Araújo* \\ Daclé V. Carvalho** \\ Gisélia R. Nunes**
}

\begin{abstract}
CASTEllanos, B. E.; ARAÚJo, C. P.; CaRValho, D. V. e NUNES, G. R. - Problemática da orientação do paciente sob controle de diurese. Rev. Esc. Enf. USP, 10(2): 183-201, 1976.
\end{abstract}

Neste estudo são verificados os tipos de orientação dados aos pacientes sob controle de diurese $e$ o que eles sabem a respeito. $O$ interesse demonstrado pelo paciente em saber do seu controle também é abordado.

\section{INTRODUÇÃO}

Em atividade profissional, como docentes de enfermagem, acompanhando alunos no campo prático, foi observado que os pacientes sob controle de diurese não sabiam explicar porque deviam guardar a urina, o que nos levou à idéia de que talvez não estivessem recebendo orientação nesse sentido. Torna-se valioso saber se os pacientes estão sendo orientados em seu controle de diurese e qual o tipo de orientação recebida, para atualizar os métodos de ensino sobre a orientação a ser dada pelos enfermeiros responsáveis por esses pacientes.

A relação do sistema urinário com os outros sistemas orgânicos é bem conhecida dentro da área da saúde, assim como a relevância da eliminação de fluidos para o controle do balanço hídrico. $O$ médico precisa da rigorosa e inteligente observação do pessoal de en-

- Docentes da Escola de Enfermagem da USP

* Docente da Escola de Enfermagem da UFMG

*** Docente da Faculdade de Enfermagem da UFPE 
fermagem, quanto às características e à medida das eliminações do paciente, e das anotações dessas observações para avaliar o tratamento (KEANE, 1969). O sucesso no controle de diurese não depende somente da eficiência da equipe de enfermagem nessas observações e anotações, mas está diretamente relacionado com o envolvimento do paciente, pois este deve ter participação ativa no seu cuidado a ele dispensado (BRUNNER, 1971; BURGERS \& BURNS, 1973).

E princípio básico da educação em saúde que os indivíduos consumidores dos préstimos da equipe de saúde estejam envolvidos com a dinâmica do tratamento, desde às primeiras fases do plano de assistência até a recuperação e manutenção do seu bem estar (HOCHBAUM, 1968). Estudos mostram que o aprendiz que participa ativamente no processo ensino-aprendizagem tem uma probabilidade maior para fazer uso dos conhecimentos adquiridos na sua progressiva educação (BEAUMONT \& WILEY, 1974). Esses conhecimentos, que para um paciente se resumem em saber $O$ que lhe sucede, onde e de que modo (HOCHBAUM, 1968), constituem parte do seu plano terapêtico; cabe ao serviço de enfermagem a responsabilidade de informá-lo sobre tal (BERNI, 1973). Um crescente número de hospitais está incluindo a educação de pacientes em seus planos de tratamento, no qual o enfermeiro desempenha papel importante. Realmente, boa técnica de enfermagem consiste, também, em ajudar o paciente a compreender e aceitar a sua doença (SHAW, 1973).

O enfermeiro, no desempenho de suas funções, assume dois papéis terapêuticos - o instrumental, ou técnico, e o expressivo, ou básico (JOHNSON \& MARTIN, 1958) - , sendo de sua responsabilidade elaborar 0 plano de cuidados para o paciente. Nesse plano a atuação na área expressiva é de sua responsabilidade e competência, e na área instrumental é de sua responsabilidade, mas pode ser delegada a outro membro da equipe de enfermagem. $O$ que se observa em campos de estágio é que, em relação ao tratamento do paciente, o enfermeiro delega aos outros membros da equipe a função de orientação e execução do controle da diurese e nem sempre avalia essas atividades, situação esta que torna difícil verificar se a orientação está sendo dada corretamente. Essa observação vem reforçar nossa hipótese de que os pacientes sob controle de diurese não estão recebendo orientação completa por parte da equipe de enfermagem. 
Função delegada ou não, o fato é que a orientação e educação do paciente não podem deixar de serem feitas (WHITEHEAL, 1970; BERNI \& FORDYCE, 1973), pois saber o que lhe sucede é um direito que the assiste (*), auxiliando-o a satisfazer a sua curiosidade. DODGE (1972), em seu estudo "What patients should be told; patients and nurses beliefs", relata haver encontrado grande número de pacientes desejosos de saber a respeito da sua doença e do seu tratamento. $\mathrm{O}$ pleno conhecimento do que lhe está acontecendo ajuda no seu equilíbrio emocional face a um stress, como é o caso da doença (SANTOS e KAMIYAMA, 1972).

Pelo exposto até aqui, podemos notar que está sendo enfatizada a participação ativa do paciente na elaboração do seu plano de assistência, visando posteriormente ao seu auto-cuidado (KREUTER, 1957; OREM, 1971).

Em vista dessas novas tendências da enfermagem, o objetivo do estudo será a verificação do que os pacientes sabem sobre o seu controle de diurese, do tipo de orientação recebida e do interesse demonstrado em receber essa orientação. Será verificada ainda a porcentagem do papel instrumental desempenhado pelo enfermeiro na orientação do paciente em controle de diurese, nos hospitais em que se fará a pesquisa.

\section{DEFINIÇÃO DE TERMOS}

Em nosso estudo empregaremos expressões que, com a finalidade de esclarecimento, serão aqui definidas:

1. Tipos de orientação

a) orientação completa - é a informação necessária para o paciente saber que está sob controle de diurese, como e porque deve ser feito esse controle.

Critérios:

saber: tomar conhecimento do fato;

* Carta dos Direitos do Paciente emitida pela Associação Americana de Hospitais, publicada no Boletim da ABEn, jan. 74 . 
como: saber onde deve guardar a urina;

porque: saber que há relação entre o controle, a sua doença $e$ a sua medicação específica.

b) orientação incompleta - quando a informação prescindir de um ou dois itens contidos na orientação completa.

2. Tipos de conhecimentos

Neste trabalho o termo "conhecimento" não terá o significado que lhe é atribuído em didática, mas por não ter sido encontrado outro termo pertinente, significará 0 que 0 paciente sabe sobre 0 seu controle de diurese. Foram convencionados dois tipos de conhecimento:

a) conhecimento completo - é aquele que abrange o conhecimento de que está sob controle de diurese, como e porque;

b) conhecimento incompleto - é aquele em que há ausência de um ou dois itens do conhecimento completo.

\section{METODOLOGIA}

\section{I - População}

1. Critérios para a seleção:

a) pacientes sob controle de diurese por indicação médica ou de enfermagem; esse critério baseia-se no fato de que alguns pacientes estão sob controle de diurese como parte do plano terapêutico médico e de enfermagem, ou apenas como parte do plano de assistência de enfermagem;

b) pacientes a partir dos quinze anos de idade, de ambos os sexos; essa delimitação de idade deve-se ao fato de quinze anos ser a idade mínima para admissão na maioria das clínicas médico-cirúrgicas;

c) pacientes conscientes e capazes de manter uma entrevista.

2. Características da população 
Ṇa população estudada foram levadas em consideração características específicas tais como:

a) Idade

ABDELLAH \& LEVINE (1957) em seu estudo "Polling patients: II - What factors affect patients opinions of their nursing care" citam que os pacientes mais jovens (até 40 anos) gostam de receber explicaçōes mais detalhadas a respeito da assistência de enfermagem que thes é prestada. Diante dessa referência, a característica idade foi levada em consideração para verificar se os mais jovens (GRUPO I desse estudo) receberam orientação completa, atendendo a esta necessidade.

TABELA I - DISTRIBUIÇÃO DE PACIENTES SOB CONTROLE DE DIURESE INTERNADOS NOS HOSPITAIS CAMPOS DA PESQUISA, POR GRUPOS ETARIOS EM RELAÇÃO AO INTERESSE DEMONSTRADO EM SABER SOBRE ESSE CONTROLE, NO PERIODO DE 5 A 24 DE MAIO DE 1975.

\begin{tabular}{|c|c|c|c|c|c|c|}
\hline Interesse & \multicolumn{2}{|c|}{ SIM } & \multicolumn{2}{|c|}{ NĀO } & \multicolumn{2}{|c|}{ TOTAL } \\
\hline etários & n. ${ }^{\circ}$ & $\%^{(\mathbf{a})}$ & n. & $\%^{(a)}$ & n..$^{\circ}$ & $\%^{(b)}$ \\
\hline $\begin{array}{l}\text { Grupo I ( } 15.40 \text { anos) } \\
\text { Grupo II (acima } \\
\text { de } 40 \text { anos) }\end{array}$ & $\begin{array}{l}65 \\
92\end{array}$ & $\begin{array}{l}67,0 \\
74,8\end{array}$ & $\begin{array}{l}32 \\
31\end{array}$ & $\begin{array}{l}33,0 \\
25,2\end{array}$ & $\begin{array}{l}97 \\
123\end{array}$ & $\begin{array}{r}44,1 \\
55,9\end{array}$ \\
\hline TOTAL & 157 & $71,4^{(b)}$ & 63 & $28,6^{(b)}$ & 220 & 100,0 \\
\hline
\end{tabular}

(a) Porcentagens calculadas sobre o númera de pacientes de cada grupo etário.

(b) Porcentagens calculadas sobre o número total de pacientes estudados.

Conforme a apresentação da TABELA I, o Grupo II demonstrou maior interesse $(74,8 \%)$, em relação ao Grupo I $(67 \%)$, em saber mais ou receber alguma informação 
sobre o seu controle de diurese. Verificando-se o interesse demonstrado pelos pacientes do Grupo I era satisfeito, quanto ao tipo de orientação recebida, conforme observação de ABDELLAH \& LEVINE, o resultado da computação mostrou que apenas um dos pacientes recebeu orientação completa, 52 receberam orientação incompleta e 12 não foram informados do controle. Isto pode levar à suposição de que os mais jovens não estão tendo sua necessidade de interesse satisfeita.

b) Grau de Instrução

TABELA II - DISTRIBUIÇĀO DE PACIENTES EM CONTROLE DE DIURESE INTERNADOS NOS HOSPITAIS CAMPOS DA PESQUISA, POR GRAU DE INSTRUÇÃO EM RELAÇĀO AO INTERESSE DEMONSTRADO EM SABER SOBRE ESTE CONTROLE, NO PERIODO DE 5 A 24 DE MAIO DE 1975.

\begin{tabular}{|c|c|c|c|c|c|c|}
\hline Interesse & \multicolumn{2}{|c|}{ SIM } & \multicolumn{2}{|c|}{ NÃO } & \multicolumn{2}{|c|}{ TOTAL } \\
\hline instrução & $\mathrm{n} .^{\circ}$ & $\%^{(a)}$ & $\mathrm{n} .^{\circ}$ & $\%^{(a)}$ & $\mathrm{n} .^{\circ}$ & $\%^{(b)}$ \\
\hline Superior & 9 & 64,3 & 5 & 35,7 & 14 & 6,4 \\
\hline $2 .^{\circ} \mathrm{Grau}$ & 6 & 54,6 & 5 & 45,4 & 11 & 5,0 \\
\hline $1 .^{\circ} \mathrm{Grau}$ & 95 & 71,4 & 38 & 28,6 & 133 & 60,4 \\
\hline Analfabeto & 47 & 75,8 & 15 & 24,2 & 62 & 28,2 \\
\hline TOTAL & 157 & $71,4^{(b)}$ & 63 & $28,6^{(\mathrm{b})}$ & 220 & 100,0 \\
\hline
\end{tabular}

(a) Porcentagens calculadas sobre o número de pacientes de cada grupo segundo o nível de instrução.

(b) Porcentagens calculadas sobre o número total de pacientes estudados.

A divisão dos pacientes estudados segundo o seu grau de instrução e o interesse por eles demonstrado em obter alguma informação, ou saber mais acerca do seu controle de diurese, encontra-se na TABELA II em que se verifi- 
cou que a variabilidade do interesse é pequena entre os níveis de instrução, encontrando-se maior interesse entre os pacientes analfabetos. Pelo fato de não ter sido feita a análise estatística da significância dos resultados obtidos com o número de pacientes encontrados em cada nível de instrução, pois estes diferem grandemente em quantidade, torna-se duvidoso afirmar a relação que o nível de instrução do paciente tem com o seu interesse.

c) Capacidade de locomoção

Em virtude de pacientes acamados terem maior ou menor grau de dependência da equipe de enfermagem, observase que a tendência é dar mais orientação ao paciente ambulante, pois este tem uma participação mais ativa no controle da diurese. A população caracterizou-se mais por pacientes ambulantes (161) do que acamados (59). A tendência observada de dar-se maior orientação aos pacientes ambulantes do que aos acamados foi comprovada, pois $44,1 \%$ dos acamados não foram informados sobre o seu controle de diurese ao passo que apenas $13,0 \%$ dos pacientes ambulantes não receberam nenhuma informação.

TABELA III - DISTRIBUIÇĀO DE PACIENTES EM CONTROLE DE DIURESE INTERNADOS NOS HOSPITAIS CAMPOS DA PESQUISA, POR CAPACIDADE DE LOCOMOÇÃO EM RELAÇÃO AO TIPO DE ORIENTAÇĀO RECEBIDA SOBRE ESTE CONTROLE, NO PERIODO DE 5 A 24 DE MAIO DE 1975.

\begin{tabular}{l|r|r|r|r}
\hline \multirow{2}{*}{$\begin{array}{c}\text { Capacidade de } \\
\text { locomoção }\end{array}$} & \multicolumn{2}{|c}{ Ambulantes } & \multicolumn{2}{c}{ Acamados } \\
\cline { 2 - 5 } Orientação & $\mathrm{n}^{\circ}$ & $\%^{\text {(a) }}$ & $\mathrm{n}^{\circ}$ & $\%^{(\mathrm{a})}$ \\
\hline Orientação completa & 7 & 4,4 & 3 & 5,1 \\
Orientação incompleta & 133 & 82,6 & 30 & 50,8 \\
Não orientados & 21 & 13,0 & 26 & 44,1 \\
\hline TOTAL & 161 & 100,0 & 59 & 100,0 \\
\hline
\end{tabular}

(a) Porcentagens calculadas sobre o número de pacientes em cada grupo segundo a capacidade de locomoção. 
Quanto ao tipo de orientação dada sobre o controle, os pacientes ambulantes receberam $87 \%$ de orientação $(4,4 \%$ destas orientações eram completas e $82,6 \%$ incompletas), ao passo que acamados que receberam $55,9 \%$ de orientação. (Veja-se TABELA III).

Cumpre notar que dos 47 pacientes que não receberam nenhuma orientação (21 pacientes ambulantes e 26 acamados), $43(91,5 \%)$ demonstraram interesse em saber sobre o controle de diurese. Dos 4 pacientes que referiram não estarem interessados, 3 eram acamados e um ambulante.

d) Primeira internação ou reinternação do paciente.

Espera-se que pacientes reinternados tragam consigo uma bagagem de experiências vividas anteriormente em ambiente hospitalar. Portanto, poderia haver variabilidade quanto ao interesse demonstrado por esses pacientes e os que estivessem sendo internados pela primeira vez em receber orientação. sobre o controle de diurese. Dos 220 pacientes, 64 estavam sendo internados num hospital pela primeira vez. Os pacientes que demonstraram interesse em receber alguma informação ou saber mais sobre o seu controle de diurese eram em número de 49 (76,5\%). Dos 156 pacientes que eram readmitidos num hospital, 88 $(56,4 \%)$ já tinham tido a experiência anterior de guardar a diurese para controle. Destes, $56(63,6 \%)$ haviam recebido alguma explicação sobre o assunto, se bem que não foi possível determinar o tipo de explicação recebida. Sobre esses pacientes, a porcentagem reinante em relação ao interesse foi de $60,7 \%$ (34 pacientes). Essa diferença de porcentagem, em relação à porcentagem do interesse demonstrado pelos pacientes admitidos no hospital pela primeira vez, leva à suposição de que o interesse dos pacientes readmitidos diminui, talvez pelo fato de trazerem consigo uma experiência já vivida em ambiente hospitalar em situações idênticas. 
II - Hospitais campos da pesquisa

Decidiu-se escolher quatro hospitais da capital do Estado de São Paulo, que receberam a denominação operacional de A, B, C e D.

Critérios para a escolha dos hospitais:

Hospitais gerais, que servem como principal campo de aprendizagem às duas faculdades e duas escolas de Enfermagem existentes na capital paulista. Nesses hospitais, foram usadas como campo de pesquisa as clínicas médica, cirúrgica e de especialidades afins (nefrolologia, urologia, neurologia, hematologia, endocrinologia), porque as afecções apresentadas pelos pacientes dessas clínicas têm maior probabilidade de estarem relacionadas com problemas que necessitam de controle de diurese.

III - Instrumento utilizado para a coleta de dados

Todas as informações pertinentes ao estudo foram colhidas através de um formulário (Anexo I) que foi elaborado pelas autoras e que consta de duas partes. A primeira foi preenchida com dados colhidos do prontuário do paciente. Consta de dados de identificação e itens que serviram para confronto com as respostas dos pacientes (diagnóstico, medicação diurética prescrita e data da prescrição do controle de diurese). A segunda parte consta de perguntas que foram feitas pelo pesquisador em entrevista com o paciente. Nessa última parte, as perguntas referemse ao conhecimento do paciente sobre o controle de sua diurese e dados outros que foram relacionados com as características já definidas da população.

Antes da sua aplicação para a coleta definitiva dos dados da população selecionada, o formulário foi submetido a um pré-teste para comprovação da sua validade, passando por modificações para melhor adaptação aos objetivos propostos.

IV - Aplicação do método

1. Foram enviados pedidos à diretoria dos hospitais escolhidos 
para o estudo, solicitando-se autorização para o uso do campo para pesquisa.

2. Foi feita a apresentação das pesquisadoras à chefe do serviço de enfermagem do hospital e à enfermeira chefe da clínica, explicando-lhes os objetivos do trabalho e fornecendo-lhes a cópia do formulário.

3. Num prazo de três semanas ( 5 a 24 de maio de 1975) foram determinados dois dias úteis consecutivos para cada hospital, a fim de ser realizada a coleta de dados.

4. No dia anterior à entrevista com o paciente, fez-se o levantamento das pessoas em controle de diurese nas clínicas escolhidas para aquele hospital. Os dados obtidos do prontuário dos pacientes foram colhidos também nesse dia.

5. No dia da entrevista com o paciente, o entrevistador apresentou-se ao mesmo, identificando-se e explicando-lhe as razões da entrevista. Uma vez obtida a aquiescência do paciente, procedeu-se à aplicação do formulário.

6. Depois de colhidos todos os dados, os seus resultados foram analisados e discutidos.

\section{DESCRIÇÃO E DISCUSSÃO DOS RESULTADDOS}

a) Quanto ao conhecimento e orientação do paciente em controle de diurese.

Dos 220 pacientes estudados, $199(90,4 \%)$ tinham conhecimento do seu controle de diurese. Um dos objetivos desse trabalho era saber desses pacientes o tipo de conhecimento que possuiam sobre a urina e seu controle e o tipo de orientação que receberam. Pela TABELA IV, nota-se que $42(21,1 \%)$ pacientes tinham conhecimento completo e $157(78,9 \%)$ conhecimento incompleto. Quanto ao tipo de orientação recebida, $10(5,0 \%)$ eram completas, $163(81,9 \%)$ incompletas e 26 $(13,1 \%)$ pacientes não receberam nenhuma orientação. 
TABELA IV - DISTRIBUIÇÃO DE PACIENTES QUE SABIAM ESTAR EM CONTROLE DE DIURESE INTERNADOS NOS HOSPITAIS CAMPOS DA PESQUISA, POR ORIENTAÇÃO EM RELAÇÃO AO TIPO DE CONHECIMENTO QUE TINHAM A RESPEITO DO CONTROLE, NO PERIODO DE 5 A 24 DE MAIO DE 1975.

\begin{tabular}{|c|c|c|c|c|c|c|}
\hline \multirow[b]{2}{*}{ Orientação } & \multicolumn{2}{|c|}{$\begin{array}{l}\text { Conhecimento } \\
\text { completo }\end{array}$} & \multicolumn{2}{|c|}{$\begin{array}{l}\text { Conhecimento } \\
\text { incompleto }\end{array}$} & \multicolumn{2}{|c|}{ TOTAL } \\
\hline & n. & $\%^{(a)}$ & n. ${ }^{\circ}$ & $\%^{(a)}$ & $n .^{\circ}$ & $\%^{(\mathrm{b})}$ \\
\hline $\begin{array}{l}\text { Orientação completa } \\
\text { Orientação incompleta } \\
\text { Não orientados }\end{array}$ & $\begin{array}{r}7 \\
34 \\
1\end{array}$ & $\begin{array}{r}70,0 \\
20,9 \\
3,8\end{array}$ & $\begin{array}{r}3 \\
129 \\
25\end{array}$ & $\begin{array}{l}30,0 \\
79,1 \\
96,2\end{array}$ & $\begin{array}{r}10 \\
163 \\
26\end{array}$ & $\begin{array}{r}5,0 \\
81,9 \\
13,1\end{array}$ \\
\hline TOTAL & 42 & $21,1^{(b)}$ & 157 & $78,9^{(b)}$ & 199 & 100,0 \\
\hline
\end{tabular}

(a) Porcentagens calculadas sobre o total de pacientes em cada tipo de orientação recebida.

(b) Porcentagens calculadas sobre o número total de pacientes estudados que sabiam estar em controle.

Pela descrição desses resultados, observou-se que das 10 orientações completas transmitidas aos pacientes, 7 (70,0\%) eram do conhecimento completo e $3(30,0 \%)$ do incompleto dos pacientes.

Foram transmitidas 163 orientações incompletas, sendo que $34(20,9 \%)$ pacientes tinham conhecimento completo sobre o controle de diurese, e $129(79,1 \%)$ conhecimento incompleto. O fato de 34 pacientes terem conhecimento completo, embora não tivessem recebido uma orientação completa, despertou a curiosidade das autoras em procurar a razão desse resultado. Pensou-se em relacionar o tipo de conhecimento (completo) desses pacientes, com a sua experiência anterior em ambiente hospitalar, sob o aspecto da eliminação urinária e seu controle. Dessa relação, verificou-se que $4(11,8 \%)$ eram admitidos pela primeira vez e 30 $(88,2 \%)$ eram reinternados, dos quais $24(80,0 \%)$ já haviam estado sob controle, e destes $16(66,6 \%)$ tinham recebido explicação acerca do assunto. As porcentagens encontradas acima levam a supor que o conhecimento completo dos 34 pacientes que receberam orientação incompleta, seja devido à vivência em condições semelhantes. 
Verificou-se que, dos 26 pacientes não orientados sobre o controle, um $(3,8 \%)$ tinha conhecimento completo, e $25(96,2 \%)$ conhecimento incompleto. Verificou-se que o paciente sem orientação mas com pleno conhecimento de seu controle de diurese era uma pessoa reintegrada várias vezes, com grau de instrução em nível colegial. Provavelmente tenham sido essas as razões de seu conhecimento completo. Das 25 pessoas restantes com o conhecimento incompleto, 16 eram reinternadas. Pelo citado anteriormente, pode-se supor que a experiência vivida em ambiente hospitalar desses pacientes tenham influenciado no referido conhecimento.

Conforme exposto até aqui, surgiu a questão de como os 26 pacientes 'que não foram orientados sabiam estar em controle de diurese. Recorreu-se a algumas observações contidas nos formulários preenchidos e notou-se que esse fato era explicado porque alguns pacientes começaram a guardar a urina em virtude de terem visto um recipiente para coleta de urina já identificado com o nome e número do leito. $O$ recipiente encontrava-se no banheiro ou ao lado de seu leito. Outras observações continham como explicação para o mesmo fato a ocorrência de pacientes serem informados do controle através de colegas de enfermaria que já estavam há mais tempo internados e já guardavam a urina.

Dos 157 pacientes que possuiam conhecimento incompleto sobre o controle de diurese foi observado que 30 sabiam somente o que deviam fazer, e 127 o que e como deviam guardar a urina. Nenhum destes sabia o porquê do controle. Voltando-se aos apontamentos, constatou-se que, dos 157 pacientes acima mencionados, apenas 3 haviam recebido orientação completa.

Segundo HOCHBAUM (1968), o paciente deve saber o que the sucede, onde e de que modo, para maior envolvimento com a dinâmica da sua assistência. Desse trabalho, surgem as indagações de como pode um paciente, desconhecendo o porquê do seu controle de diurese e não sendo orientado tão pouco para tal, participar do seu plano terapêutico? Será que a assistência prestada aos pacientes está sendo planejada conforme as tendências atuais de enfermagem que visam à educação do paciente para seu auto cuidado? (OREM, 1971). Respostas a essas perguntas merecem investigações futuras. 
Vinte e um pacientes que estavam com a prescrição de controle de diurese (o tempo da data da prescrição até a entrevista com o paciente variava de 1 a 30 dias) não tinham conhecimento de tal assunto. Pelas respostas registradas nos formulários, foi verificado que nenhum deles recebeu orientação.

b) Quanto ao interesse demonstrado pelo paciente sobre o controle de diurese.

Para determinar o interesse demonstrado pelo paciente sobre o seu controle de diurese, foi formulada uma pergunta dirigida àqueles que tinham conhecimento desse assunto e que gostariam de saber mais alguma coisa sobre o mesmo, e outra para aqueles que não haviam sido orientados, mas gostariam de receber alguma informação.

De todos os 199 pacientes que sabiam estar sob controle, $134(67,3 \%)$ demonstraram interesse em saber mais a respeito.

Foi-lhes perguntado o que gostariam de saber, e o resultado segue abaixo.

Gostariam de saber:

- porque guardam a urina e o que fazem com ela (71 pacientes);

- tudo o que seja relacionado com a urina e a doença (41 pacientes);

- que exames fazem com a urina, e seus resultados (10 pacientes);

- as características da urina e o seu significado (6 pacientes).

Houve 6 pacientes que demonstraram interesse, mas não souberam explicar o que gostariam de saber além do que já sabiam.

Foi encontrada incoerência entre as respostas dadas pelos pacientes que sabiam estar sob controle e não demonstravam interesse 
em saber mais alguma coisa sobre ele. Sessenta e um $(93,8 \%)$ dos 65 declararam que a orientaçāo deveria ser dada a todos os pacientes que estivessem em controle de diurese.

A porcentagem de interesse demonstrado em receber alguma orientação, por parte dos doentes que desconheciam estar sob controle (21), foi de $100 \%$.

De toda a população (220), a porcentagem de pacientes que demonstrou interesse foi de $70,4 \%$. Isso confirma o que DODGE (1972) declara ter encontrado em seu estudo - "grande número de pacientes desejosos de saber a respeito de sua doença e tratamento". A existência desse interesse foi reforçada quando 109 pacientes responderam que costumam examinar a sua urina. A característica mais evidente observada pelos pacientes foi a cor da eliminação urinária, seguida da existência de sedimentos e da quantidade eliminada.

Indagou-se de todos os pacientes entrevistados a sua opinião sobre quem deveria receber as informações do controle de diurese. Cento e cinquenta e seis $(70,9 \%)$ achavam que essa informação deveria ser extensiva a todos os pacientes que estavam sob controle. Quarenta e nove pacientes $(22,2 \%)$ achavam que as informações deveriam ser dadas somente aos que as pedissem, e $15(6,8 \%)$ deram as mais variadas respostas. Por esses resultados, nota-se que há concordância nas porcentagens entre a opinião emitida e o interesse em receber alguma orientação.

c) Quanto ao papel instrumental desempenhado pelo enfermeiro.

O último objetivo do trabalho era verificar a porcentagem do papel instrumental desempenhado pelo enfermeiro na orientação do paciente sob controle de diurese. No estudo, foi constatado que a primeira informação ao paciente sobre seu controle foi dada pelo enfermeiro (51), pelo médico (41), pelo aluno de enfermagem (5), pelo auxiliar de enfermagem (32), pelo atendente (24) e 20 pacientes não se lembravam da pessoa que lhes informou. Segundo respostas concretas dos pacientes, as informações dadas pelo enfermeiro atingiram a porcentagem de $29,5 \%$ sobre o total de 173 orientações dadas.

A curiosidade das autoras foi despertada para saber o tipo de orientação dada pelo enfermeiro aos pacientes: 44 eram incompletas 
e 7 completas. Isso pode levantar a suposição de que a maioria dos enfermeiros não está dando uma orientação completa ao paciente.

Ao ser definido esse último objetivo do estudo, a preocupação se dirigiu no sentido de saber o quanto o enfermeiro participa diretamente na orientação do paciente sob controle de diurese. Uma vez que as orientaçōes dadas pelo enfermeiro são na maioria incompletas, como pode este profissional esperar a participação ativa do paciente no seu plano de assistência?

Com exceção das orientações fornecidas pelo médico, o enfermeiro é o responsável pela supervisão e a avaliação dos papéis delegados aos outros membros da equipe de enfermagem, quanto às orientações a serem prestadas aos pacientes. Será que esta supervisão e avaliação existem na realidade?

Antes que surjam respostas concretas a essas indagações, torna-se necessário saber se a enfermagem dos hospitais pesquisados segue as novas tendêneias profissionais no que diz respeito à orientação do paciente para seu auto cuidado.

\section{CONCLUSOES}

1. Entre os pacientes estudados, $157(78,9 \%)$ não sabem porque estão guardando a urina, sendo que 3 receberam orientações completas sobre o controle, 129 orientações incompletas, e 25 não foram orientados sobre o assunto.

2. Do total de pacientes, encontrou-se a porcentagem de $70,4 \%$ de interesse demonstrado pelos entrevistados em saber ou conhecer mais a respeito de seu controle de diurese.

3. A porcentagem do papel instrumental desempenhado pelo enfermeiro na orientação do paciente sob controle de diurese nos hospitais campos da pesquisa foi de $29,5 \%$. 


\section{RECOMENDAÇÃO:}

Que enfermeiros e alunos de enfermagem dediquem mais tempo em dar, supervisionar e avaliar as orientações fornecidas aos pacientes sobre o porquê do controle de diurese, para que estes participem mais ativamente do seu plano assistencial.

CASTEllanos, B. E.; ARAÚJO, C. P.; CaRValho, D. V. e NUNES, G. R. - The problematic of orientating patients under diuresis-control. Rev. Esc. Enf. USP 10(2): 183-201, 1976.

ABSTRACT: The study reveals the kind of orientation given to patients under diuresis control; verifies what they know about it and find out the percentual of interest shown by the patients in getting more informations regarding the control.

\section{REFERENCIAS BIBLIOGRAFICAS}

ABDELLAH, F. G. \& LEVINE, E. - Polling patients and personnel: II - What factors affect patients' opinions of their nursing care? Hospitals, 31: 61-4, nov. 16, 1957.

ASSOCIAÇÃO AMERICANA DE HOSPITAIS - Carta dos direitos do paciente. Informativo $A B E n$, jan., 1974.

BEAUMONT, E. \& WILLEY, L. - Innovations in nursing. Nursing' 74, 3 (3): 15-7, mar. 1974.

BERNI, R. \& FORDYCE, W. E. - Behavior modification and the nursing process. Saint Louis, Mosby, 1973.

BRUNNER, L. S. et all - Enfermeria medicoquirurgica. $2 .^{\circ}$ ed. México, Interamericana, 1971.

BURGESS, A. C. \& BURNS, J. - Why patients seek care. Amer J Nurs, 73 (2): 314-6, feb. 1973.

DODGE, J. S. - What patients should be told: patients' and nurses' beliefs. Amer J Nurs, 72 (10): 1852-4, oct. 1972. 
HOCHBAUM, G. M. - A participação do consumidor no planejamento de saúde: algumas consideraçōes teóricas; Trad. de Nelly M. F. Candeias. Trabalho apresentado em Genebra, OMS, 1968 (mimeografado).

JOHNSON, M. M. \& MARTIN, H. W. - A sociological analysis of the nurse role. Amer J Nurs, 58 (3): 373-7, mar. 1958.

KAMIYAMA, Y. - $O$ doente hospitalizado e sua percep̧̧ão quanto à prioridade de seus problemas. São Paulo, 1972. (Tese - Escola de Enfermagem da Universidade de São Paulo).

KEANE, C. B. - Essentials of nursing: a medical surgical text for practical nurses, 2nd. ed. Philadelphia, Saunders, 1969.

KREUTER, F. R. - What is good nursing care? Nurs Outlook, 5 (5): 302-4, may 1957.

OREM, D. E. - Nursing: concepts of practice. New York, McGrawHill, 1971.

SANTOS, A. L. V. - Contribuição ao estudo da comunicação paciente - equipe de enfermagem relativa à medicação. São Paulo, 1972. (Tese - Escola de Enfermagem da Universidade de São Paulo).

SHAW, J. S. - New Hospital commitment: teaching patients how to live with illness and injury. Mod Hosp. 121 (10): 99-102, oct. 1973.

WHITEHEAD, S. L. - Nursing care of the adult urology patient. New York, Appleton-Century-Crafts, 1970. 


\section{ANEXO I}

FORMULÁRIO

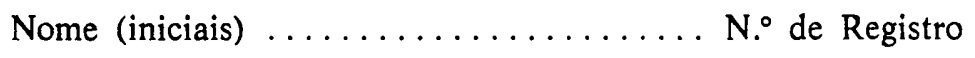

Hospital

Clínica

Idade

Sexo

Grau de instrução

Diagnóstico

Medicação diurética prescrita

Data da prescrição do controle de diurese

1. Esta é a primeira vez que o(a) senhor(a) fica internado(a) no hospital?

$\square \operatorname{SIM} \square$ NÃO

CASO NÃO:

a) $\mathrm{O}$ (a) senhor(a) da(s) outra(s) vez(es) precisou guardar toda a urina do dia para medir?

$\square \quad$ SIM — Recebeu alguma explicação sobre o controle da urina?

$\square \operatorname{SIM}$

$\square \quad N \AA ̃ O$

$\square \quad N A ̃ O$

2. $\mathrm{O}$ (a) senhor(a) pode ir ao banheiro para urinar?

$\square$ SIM $\square$ NÃO

3. Neste hospital, o(a) senhor(a) está guardando a sua urina?
$\square$ SIM
$\square \quad$ NÃO

CASO SIM:

a) Como guardar a urina? 
b) Por que está guardando a urina?

c) $O$ (a) senhor(a) costuma examinar a sua urina?

$\square \quad \operatorname{SIM}-\mathrm{O}$ que olha na urina?

$\square$ NÃO

d) Há mais alguma coisa que o(a) senhor(a) gostaria de saber em relação ao controle da urina?

$\square$ SIM - O que?

$\square \quad N A ̃ O$

4. Alguém lhe informou que o(a) senhor(a) está em controle de diurese? $\square$ SIM - Qual o conteúdo da informação? (incluindo como e porque)

Quem foi que lhe informou? (investigar a função da pessoa citada)

$\square$ NÃO - Tem interesse em receber alguma explicação? $\square$ SIM $\square$ NÃO

5. $O$ (a) senhor(a) acha que as informações sobre o controle da urina devem ser dadas para:

$\square$ Todos os pacientes que guardam urina?

$\square$ Para os pacientes que pedirem informação?

$\square$ Outros? (especificar)

Observações

Data da entrevista

Nome do entrevistador 